\title{
Results of external beam radiotherapy for diffuse choroidal hemangiomas in Sturge-Weber syndrome
}

\author{
Matthieu Randon ${ }^{1} \cdot$ Christine Lévy-Gabriel $^{1} \cdot$ Rachid Abbas $^{2,5} \cdot$ Rémi Dendale $^{3}$ - Livia Lumbroso ${ }^{1}$ • \\ Laurence Desjardins ${ }^{1} \cdot$ Nathalie Cassoux ${ }^{1,4}$
}

Received: 9 July 2017 / Revised: 4 October 2017 / Accepted: 15 November 2017 / Published online: 6 February 2018

(c) The Royal College of Ophthalmologists 2018

\begin{abstract}
Purpose The Sturge-Weber Syndrome (SWS) is a phacomatosis which include facial nevus flammeus, glaucoma, diffuse choroidal hemangioma, and leptomeningeal hemangiomatosis. External beam radiotherapy (EBRT) using photons was used to treat retinal detachment. We investigate the anatomical and functional results in a long-term basis.

Methods Retrospective review of SWS patients treated by EBRT (20 Gy in 10 fractions) for an exudative diffuse choroidal hemangioma. Visual acuity, B-scan tumor thickness, size of retinal detachment, intra-ocular pressure, and hypotonic treatment were collected before EBRT, 1 year after, and at the latest news.

Results Twenty-five patients (26 eyes) were treated between 2001 and 2014. Retinal detachment including the macula was found among twenty-six eyes before treatment. The average follow-up time was 47 months. The mean tumor thickness was initially $4.5 \mathrm{~mm}, 2.8 \mathrm{~mm}$ at first year, and $2.7 \mathrm{~mm}$ at the last visit. The retina was reattached at the last visit for all eyes except two. The visual acuity was stable or better for 20 eyes $(p=0.02)$. Four patients developed mild cataract during the followup.

Conclusion EBRT using $20 \mathrm{~Gy}$ in 10 fractions is efficient, decreases tumor thickness, reattaches the retina, and stabilizes visual acuity. In the long term, retinal reattachment allows ocular conservation by preventing phthisis bulbi.
\end{abstract}

\section{Introduction}

The Sturge-Weber syndrome (SWS) is a rare, sporadic neuro-oculocutaneous disorder. Clinical features include facial nevus flammeus (port-wine stain), buphthalmos, epilepsy, mental retardation, and hemiplegia. One of the most frequent ocular manifestations of this syndrome is a diffuse choroidal hemangioma (DCH). In SWS, port-wine stains of the eyelids (upper more common than lower), bilateral distribution of the birthmark, and unilateral port-

Matthieu Randon

matthieu.randon@gmail.com

1 Ocular Oncology, Institut Curie, Paris, France

2 Biostatistics Unit, Gustave Roussy, Villejuif, France

3 Radiation Oncology, Institut Curie, Paris, France

4 School of Medicine Paris V Descartes, Université PSL Paris, France

5 Team-Oncostat, Paris-Sud University, Villejuif, France wine stains involving the upper and lower face are associated with a greater likelihood of having eye and/or central nervous system (CNS) complications [1]. CNS involvement includes ipsilateral leptomeningeal hemangiomatosis presenting with seizures in about $80 \%$ of cases [2]. Glaucoma, due to either angle maldevelopment or raised episcleral venous pressure, is observed in 48 to $71 \%$ [3].

SWS occurs almost entirely sporadically and with equal frequency in both sexes. The genetic, environmental, or prenatal factors resulting in the disorder are not perfectly known. The localized abnormalities of blood vessel development and function affecting the facial skin, eye, and brain suggest a developmental disruption occurring in the first trimester of pregnancy [4]. During early stage 2 to 3 of vascular development in the first trimester, the primitive vascular system divides into the external portion that feeds and drains the facial skin and scalp, a middle portion investing the meninges, and the deep portion that feeds and drains the brain [5]. A somatic mosaic mutation involving these embryologic tissues has been suggested as one mechanism for the occurrence of SWS [6]. 


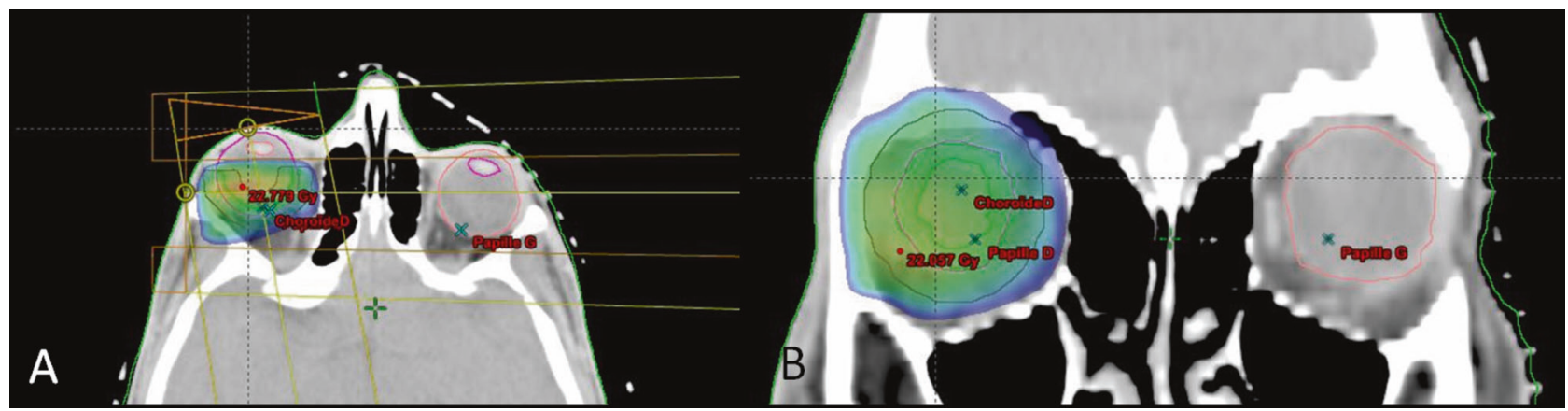

Fig. 1 Dosimetry before choroidal external radiotherapy. a Axial view with dosimetry of the right eye. b Frontal view with dosimetry of the right eye posterior pole

In most of cases, DCHs are evident clinically as diffuse red ("tomato-ketchup") fundus appearance with choroidal thickening and increased tortuosity of retinal vessels. These DCH often involve more than one-half of the fundus area. Sometimes chroroidal hemangiomas of SWS may be more subtle with lack of well-defined borders. Vision loss may result from refractive error and foveal distortion, as well as an exudative retinal detachment [7]. Ultrasonography, fluorescein angiography, optical coherence tomography, and magnetic resonance imaging can be useful in confirming the diagnosis. Fluorescein angiography demonstrates a characteristic early diffuse speckled choroidal flush. Bscans are notable for solid highly echogenic lesions with diffuse choroidal thickening, whereas A-scans exhibit high internal reflectivity.

Although these hemangiomas are congenital, exudative retinal detachment may develop at any time through adolescence, with mean age of onset of 8 years. They are observed in approximately half of patients with SWS. Clinicopathologic correlations have suggested that in eyes presenting retinal detachment there is often a localized nodular component to the overall diffuse lesion.

In recent years, there have been several reports of using low-dose (12-20 Gy) external beam radiotherapy (EBRT) with photons [8-11]. After treatment, these patients had improved visual acuity (VA), tumor regression, and resolution of subretinal fluid [8]. The primary goal of this study is to evaluate the results of EBRT in terms of tumor's thickness, retinal reattachment, VA, and side effects with a bigger amount of patients. The secondary goal is to study the irradiation effect on ocular hypertension and glaucoma.

\section{Methods}

We have achieved a retrospective study of DCHs treated by EBRT at the Institut Curie in Paris between 2001 and 2014. All cases were diffuse hemangioma in the context of SWS; circumscribed lesions were excluded from this study. All patients presented symptoms related to macula off retinal detachment: loss of VA in every cases, sometimes associated with metamorphopsias, photopsia, or scotoma. The diagnosis was based on the clinical appearance of the tumor on ocular fundus examination, correlated with ultrasound and angiography findings. Collected data included demographic (age, gender), presence of CNS angioma, presence of seizure, skin angioma localization, initial ocular features (VA in log of the minimum angle of resolution (logMAR), interval between onset of symptoms and diagnosis, intraocular pressure (IOP), history of glaucoma, presence of cataract, treatment received), initial tumor features (presence of retinal detachment and size, retinal exudation, tumor thickness on standard ocular ultrasound), irradiation parameters. The tumor thickness was evaluated by an ophthalmolgist at the bigger thickness.

All patients received EBRT with high energetic photons. Patients were treated in a supine position on a couch. Clinical target volume (CTV) included the choroid volume. A total dose of $20 \mathrm{~Gy}$ ( $2 \mathrm{~Gy}$ per fraction) was routinely delivered on CTV by lateral, or lateral and anterior beam. The choroid irradiation was done using a lens-sparing technique (Fig. 1).

Treatment and clinical data were reviewed retrospectively and recorded in our database at 1 year and at last control. A complete functional assessment was performed with estimation of VA, IOP, glaucoma treatments, and systematic search for the following post-radiation sequelae (palpebral sequelae, dry eye syndrome, keratitis, posterior synechiae, uveitis, iridial neovascularization, hyphema, cataract, radiation retinopathy). The clinical tumor response and the ultrasound tumor thickness were recorded at 1 year and at the last exam. A recurrence was defined as a treatment response first, then a tumor progression associated with a retinal detachment.

Statistical analysis was performed on demographic data, tumor data, and follow-up data. A $\chi^{2}$ test was used to compare two percentages and a Student's $t$ test was used to compare two means. For repeated outcome measures, paired 
$t$ test and Mc Nemar's $\chi^{2}$ tests were used. Quantitative variables are presented with mean and range. All tests were two sided with a $p=0.05$ significance level.

\section{Results}

\section{Demographic results}

The mean age of the patients was 15 years (4-41). All of them had a nevus flammeus, 14 on the right side, 7 on the left side, and 4 with bilateral involvement. Ten patients had a CNS angioma, complicated with seizure in 5 cases. No one had mental retardation preventing the radiotherapy. Two eyes had a previous laser treatment (PDT) and one underwent a vitrectomy. A total of twenty-six eyes exhibited a DCH as part of the SWS (25 patients but one had bilateral DCH, 11 males/15 females) were irradiated with photon beams (Table 1). The mean follow-up time was 47 months (range from 8 months to 11 years).

\section{Anatomic results}

Each patient had a retinal detachment involving or threatening the fovea, 16 in the right eye and 10 in the left eye, 5 had a bullous one (Fig. 2). Mean tumor thickness was 4.6 $\mathrm{mm}(3-7.5 \mathrm{~mm})$ at baseline (Table 2). All eyes were preserved at last follow-up. Shrinkage of the tumor mass was seen in 25 eyes $(96 \%)$ and confirmed by standardized ultrasound which showed a tumor thickness decrease from 4.6 to $2.8 \mathrm{~mm}$ in 1 year $\left(p=10^{-8}\right.$, paired Student's $t$ test) and $2.7 \mathrm{~mm}$ at the final exam (Fig. 3a). The reattachment of the retina was achieved in 21 eyes $(80 \%)$ at the first year, and in 24 eyes (92\%) at the last exam, $\left(p=10^{-8)}\right.$. Tumor shrinkage followed by retinal reattachment were usually observed between the third and the fourth month after EBRT. Two local recurrences were detected. The first one appeared 5 years after irradiation, and was treated with a second EBRT with the same protocol. The results were

Table 1 Population description before the choroidal hemangioma treatment

\begin{tabular}{ll}
\hline Retinal detachment & $n=26$ \\
Mean age and range (years) & $15(4-41)$ \\
Ratio (M/F) & 0.73 \\
CNS angioma & $38 \%$ \\
Mean tumor thickness (mm) and range & $4.6(3-7.5)$ \\
VA better than + 1 logMAR & $n=14$ \\
History of glaucoma & $n=14$ \\
Mean IOP among glaucoma patients (mmHg) and range & $20(10-33)$ \\
\hline
\end{tabular}

CNS central nervous system, IOP intra-ocular pressure, logMAR log of the minimum angle of resolution good 6 years after (tumor thickness went from 4 to $2 \mathrm{~mm}$ ). The second one had a mild recurrence 7 years after treatment, and its follow-up is still running.

\section{VA evaluation}

The median time of vision loss was 9 months (range from 7 days to 156 months) (Table 2). The majority (17 patients, $65 \%$ ) had a vision loss for 6 months or more. VA was better than or equal to $1 \log$ MAR in 17 eyes and upper than 1 $\operatorname{logMAR}$ in 9 eyes. The VA at first year improved by 1 or more ETDRS lines in 13 eyes (50\%) and was unchanged or deteriorated in 13 eyes $(50 \%)(p=0.02)$. At the final exam, VA improved by 1 or more ETDRS lines in 13 eyes (50\%) and was unchanged or deteriorated in 13 eyes $(50 \%),(p=$ 0.02) (Fig. 3b). The final VA seems to be better if time lag between first symptoms and consultation is low (less than 6 months) ( $p=0.12$ at Wilcoxon's test) (Fig. 3b).

\section{IOP evolution}

Fourteen patients had glaucoma, seven underwent a medical treatment, three a surgical one (trabeculectomy), and four had both. The mean IOP in those patients was $20 \mathrm{mmHg}$ (10-33) at baseline. Three patients with medication before irradiation were authorized to stop it 1 year after. The mean IOP of patients with a history of glaucoma was $18.5 \mathrm{mmHg}$ (7.5-33) at final exam (comparison of the first and the final IOP by Student's $t$ test, $p=0.31$ ).

Twelve patients had no history of glaucoma and the mean IOP was $15 \mathrm{mmHg}$ (10-20). Two patients without history of glaucoma required anti-glaucomatous medication after irradiation. The mean IOP of patients without a history of glaucoma was $13.8 \mathrm{mmHg}$ at final exam (10-20) (comparison of the first and the final IOP by Student's $t$ test, $p=$ $0.31)$.

\section{Side effects}

As acute side effects (within 15 days after irradiation), we found five (19\%) eyelid erythema, seven (27\%) conjunctival hyperemia, and four $(15 \%)$ orbital pain. As chronic side effects, there were four $(15 \%)$ mild cataract, developed at least 3 years after the treatment. Three cataracts appeared among children and one among adults. Neither keratitis nor radiation retinopathy were seen.

\section{Discussion}

The results of this study demonstrate the benefit of low-dose ocular irradiation for resorption of the subretinal fluid over symptomatic choroidal hemangiomas. As a result of the 




Fig. 2 Example of a patient with a choroidal hemangioma treated by external irradiation. a Fundus photography of a retinal detachment before irradiation. b Fundus photography of a retinal reattachment 1 year after irradiation. c Hemangioma ultrasound complicated by an

proximity of most choroidal hemangiomas to the foveal region, a prompt decrease of VA due to exudative retinal detachment is often an early symptom. The associated damage to the foveal photoreceptor layer is comparably small when the detachment of the retina is of short duration. As a consequence of persisting exudation, degenerative changes in the foveal retina, secondary fibrous metaplasia, and cystoid macular edema lead to irreversible functional loss. Irrespective of treatment option, a stable reattachment of the fovea would probably allow the most desirable form of restoration of visual function.

Institut Curie-Proton therapy center had a clinical experience of proton beam irradiation for circumscribed choroidal hemangiomas [12]. The proton protocol was different from the photon's one. It used a fixed horizontal proton beam line and patients were sat on a robot chair. The dose delivered was $20 \mathrm{~Gy}$ in 4 fractions of $5 \mathrm{~Gy}$ on a tumor volume surrounded by a safety margin of $2.5 \mathrm{~mm}$. For inferior retinal detachment before irradiation. d Hemangioma ultrasound 1 year after irradiation showing a retinal reattachment and tumor shrinkage

diffuse $\mathrm{CH}, \mathrm{CTV}$ corresponded to the entire choroid volume. The protons would have irradiated almost the totality of the eye volume. We have considered that such treatment (high dose per fraction (5 Gy) and great irradiated eye volume) could lead to eye complications. We decided that all diffuse $\mathrm{CH}$ would be treated with a conventional radiotherapy approach: $20 \mathrm{~Gy}$ in 10 fractions of $2 \mathrm{~Gy}$ with photons beams. Chan et al. [13] and Zeisberg et al. [14] had reported eye complications after proton beam irradiation of diffuse $\mathrm{CH}$, delivering 15 to $30 \mathrm{~Gy}$ and $20 \mathrm{~Gy}$ in 4 fractions, respectively.

In our study, an irradiation of the dose of $20 \mathrm{~Gy}$ in 10 fractions of $2 \mathrm{~Gy}$ is not tumor destructive but lead to a complete resorption of the subretinal fluid in the majority of cases associated with a reattachment of the fovea achieved in all cases. The biological effect of the low-dose radiation on $\mathrm{CH}$ is unknown, especially since there are no histological description available nor are there systematic studies 


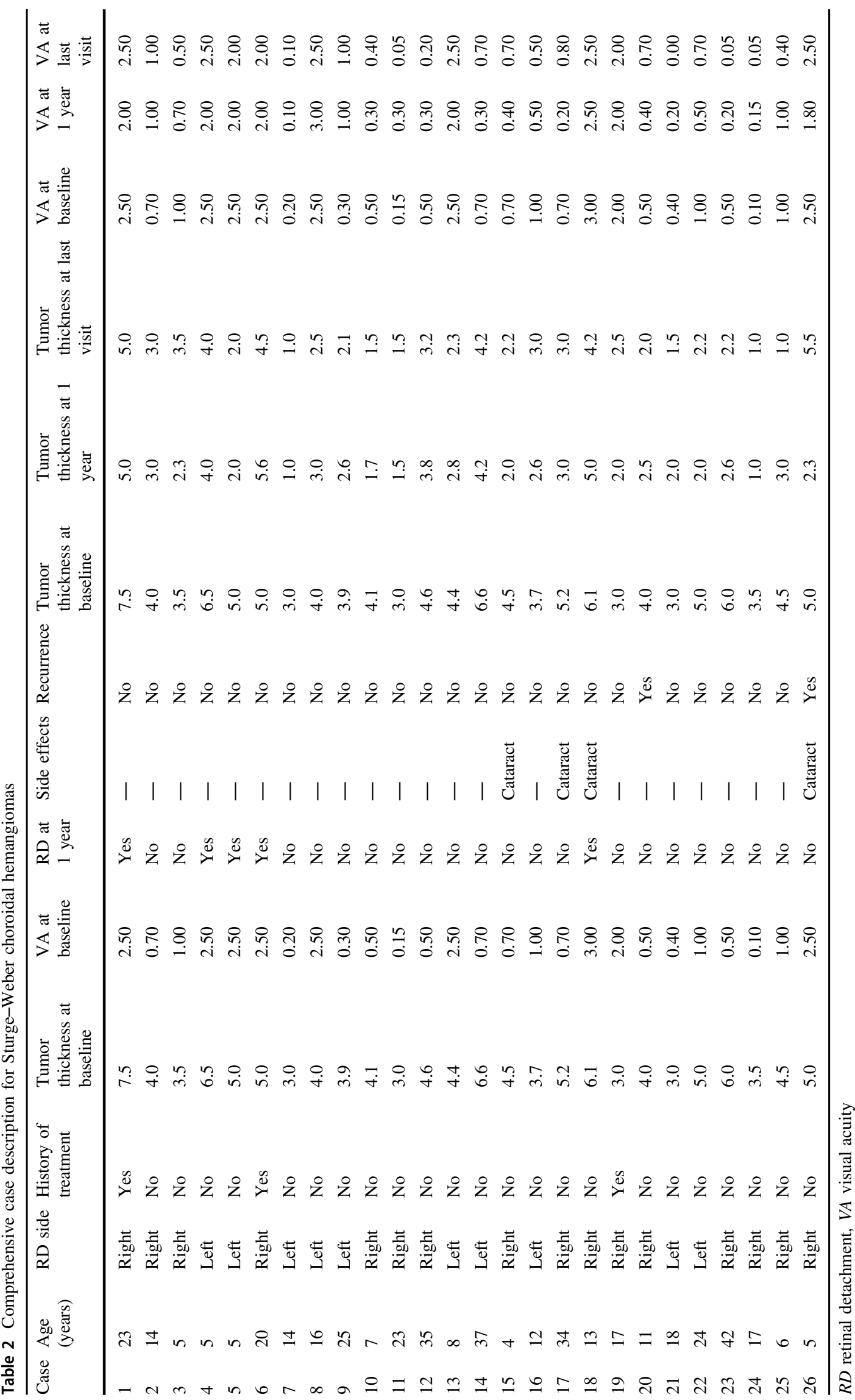




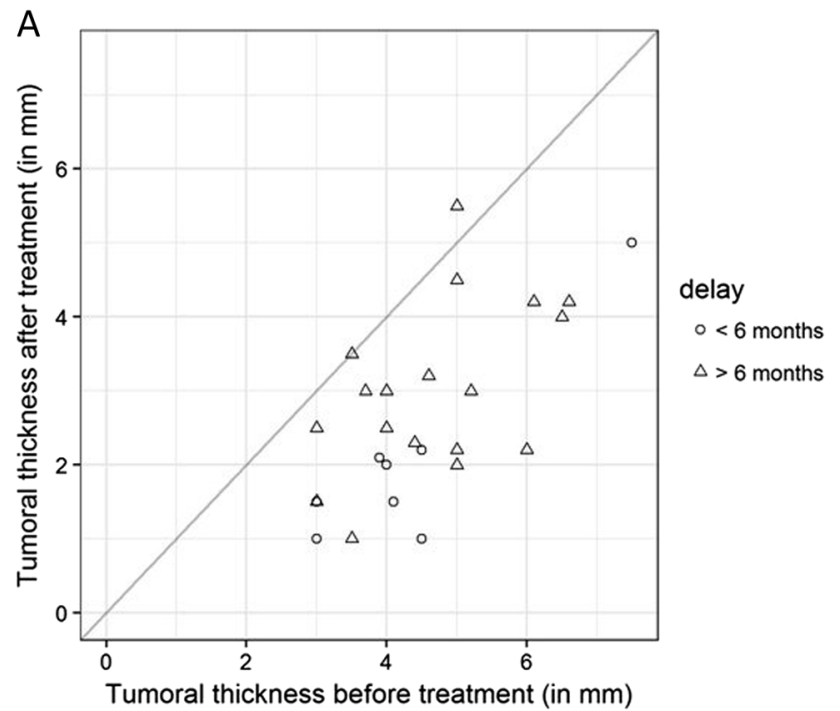

Fig. 3 a Scatter plot of tumor thickness evolution correlated with the time lag (before or after 6 month) between the first symptoms and the treatment. b Scatter plot of visual acuity evolution correlated with the time lag (before or after 6 month) between the first symptoms and the

of the radiation effects on benign vascular tumors [15]. Posterior segment complications are also less likely because of the low dose of radiation. It must not be overlooked that radiation exposure during childhood increases lifetime risk of developing secondary cancers [16].

This is the largest series, while the other four main publications are those by Schilling et al. (15 cases) [9], Madreperla et al. (5 cases) [8], Rumen et al. (5 cases) [10], and Scott et al. (4 cases) [11]. In 1997 Schilling et al. [9] reported their results of a comparable technique of external irradiation on 15 eyes with diffuse $\mathrm{CH}$ and bullous serous retinal detachment as a part of the SWS. Their results for diffuse $\mathrm{CH}$ were comparable with those of our study in the fact that complete resolution of subretinal fluid was achieved, although there was no substantial improvement of VA.

The use of oral propranolol hydrochloride has been described for infantile orbital and periorbital hemangioma. Recently, Léauté-Labrèze et al. [17] observed that systemic propranolol could inhibit the growth of infantile orbital hemangioma lesions. Krema et al. [18] reported the failure of oral propranolol for exudative retinal detachment in DCH associated with SWS.

Theoretically, PDT has ideal properties for treating circumscribed choroidal hemangioma as it can offer sitespecific tumor destruction while sparing the overlying retina and retinal vasculature, especially if the retina is detached over the tumor. This treatment has not been used for treating a large amount of patients [19].

The mechanism of glaucoma in SWS may change with age. In children $<2$ years old, goniodysgenesis is noted,

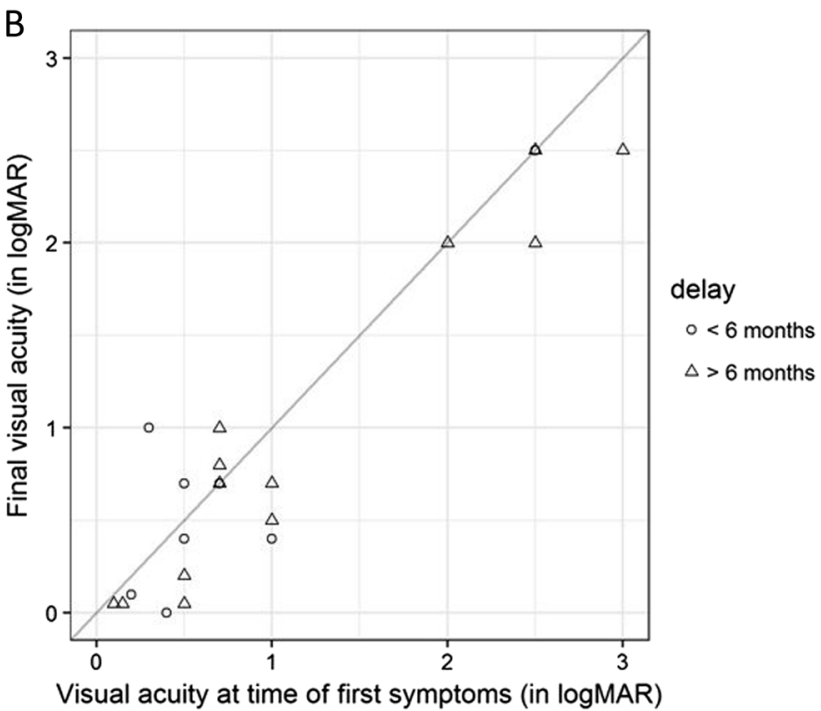

treatment. The final visual acuity seems to be better if time lag between the first symptoms and the consultation is low ( $<6$ months) $p=0.12$ at Wilcoxon's test

whereas glaucoma with an onset later in childhood may be due to high episcleral venous pressure (EVP) [20, 21]. Increased EVP causes a resistance to outflow from the anterior chamber [22]. Phelps and Armaly measured elevated EVP in 11 of 12 eyes of patients with SWS, facial port-wine mark, and glaucoma [23]. In multiple studies of patients with glaucoma secondary to SWS, the majority of children required additional procedures after their initial goniotomy or trabeculotomy [24]. Port-wine marks are known to grow progressively due to vessel hypertrophy and dilation with age [25]. It is possible that this pattern of vascular hypertrophy is also occurring at the level of the episcleral veins, resulting in later onset of elevated EVP [21]. Treatments for SWS-related glaucoma include both topical medication and surgical intervention. The most wellstudied topical medication is latanoprost (Xalatan, Catalent Pharma Solutions (part of Pfizer), Woodstock, Illinois) [26, 27]. The mechanism of action of increasing uveoscleral outflow theoretically bypasses the problems of EVP affecting the natural aqueous drainage process. We hypothesize that uveo-scleral outflow could be increased by diminishing hemangioma volume. The effect of irradiation on IOP stays unclear. Irradiation could increase uveo-scleral outflow by decreasing uveal pressure. An IOP reduction was noted but not statistically significant and many biases such as adjunction of anti-glaucomatous medication make interpretation difficult.

In this study, we have reported that EBRT by photons and or electrons beam, giving low total dose $(20 \mathrm{~Gy})$ and low dose per fractions ( $2 \mathrm{~Gy}$ ) represents an efficient treatment for diffuse $\mathrm{CH}$. A critical factor in a successful 
outcome would be the early commencement of the radiotherapy upon the first presentation of subjective signs of deterioration. Irreversible loss of eye function can be anticipated when symptoms are of long duration.

\section{Summary}

\section{What was known before}

- The external radiotherapy could reduce the tumoral volume, reattach the retina, and preserve the VA of Sturge-Weber choroidal hemangiomas.

\section{What this study adds}

- Twenty-six eyes were treated with $20 \mathrm{~Gy}$ in 10 fractions.

- We observed a $51 \%$ shrinkage of the tumor and a vision stabilization.

\section{Compliance with ethical standards}

Conflict of interest The authors declare that they have no competing interests.

\section{References}

1. Tallman B, Tan OT, Morelli JG, et al. Location of port-wine stains and the likelihood of ophthalmic and/or central nervous system complications. Pediatrics. 1991;87:323-7.

2. Sujansky E, Conradi S. Outcome of Sturge-Weber syndrome in 52 adults. Am J Med Genet. 1995;57:35-45.

3. Sujansky E, Conradi S. Sturge-Weber syndrome: age of onset of seizures and glaucoma and the prognosis for affected children. $\mathbf{J}$ Child Neurol. 1995;10:49-58.

4. Maiuri F, Gangemi M, Iaconetta G, et al. Sturge-Weber disease without facial nevus. J Neurosurg Sci. 1989;33:215-8.

5. Plate KH. Mechanisms of angiogenesis in the brain. J Neuropathol Exp Neurol. 1999;58:313-20.

6. Happle R. Lethal genes surviving by mosaicism: a possible explanation for sporadic birth defects involving the skin. J Am Acad Dermatol. 1987;16:899-906.

7. Anand R, Augsburger JJ, Shields JA. Circumscribed choroidal hemangiomas. Arch Ophthalmol. 1989;107:1338-42.

8. Madreperla SA, Hungerford JL, Plowman PN, et al. Choroidal hemangiomas: visual and anatomic results of treatment by photocoagulation or radiation therapy. Ophthalmology. 1997;104:1773-8.
9. Schilling H, Sauerwein W, Lommatzsch A, et al. Long-term results after low dose ocular irradiation for choroidal haemangiomas. Br J Ophthalmol. 1997;81:267-73.

10. Rumen F, Labetoulle M, Lautier-Frau M, et al. Sturge-Weber syndrome: medical management of choroidal hemangiomas. J Fr Ophtalmol. 2002;25:399-403.

11. Scott TA, Augsburger JJ, Brady LW. et al. Low dose ocular irradiation for diffuse choroidal hemangiomas associated with bullous nonrhegmatogenous retinal detachment. Retina. 1991;11:389-93.

12. Levy-Gabriel C, Rouic LL, Plancher C, et al. Long-term results of low-dose proton beam therapy for circumscribed choroidal hemangioma. Retina. 2009;29:170-75.

13. Chan RV, Yonekawa Y, Lane AM. et al. Proton beam irradiation using a light-field technique for the treatment of choroidal hemangiomas. Ophthamologica. 2010;224:209-16.

14. Zeisberg A, Seibel I, Cordini D, et al. Long-term (4 years) results of choroidal hemangioma treated with proton beam irradiation. Graefes Arch Clin Exp Ophthalmol. 2014;252:1165-70.

15. Plowman PN, Harnett AN. Radiotherapy in benign orbital disease. I: Complicated ocular angiomas. $\mathrm{Br} \quad \mathrm{J}$ Ophthalmol. 1988;72:286-8.

16. Ron E. Ionizing radiation and cancer risk: evidence from epidemiology. Pediatr Radiol. 2002;32:232-7.

17. Léauté-Labrèze $\mathrm{C}$, Dumas de la Roque $\mathrm{E}$, Hubiche T. Propranolol for severe hemangiomas of infancy. $N$ Engl $J$ Med. 2008;358:2649-51.

18. Krema H, Yousef YA, Durairaj P, et al. Failure of systemic propranolol therapy for choroidal hemangioma of Sturge-Weber syndrome: a report of 2 cases. JAMA Ophthalmol. 2013;131:681-3.

19. Tsipursky MS, Golchet PR, Jampol LM. Photodynamic therapy of choroidal hemangioma in Sturge-Weber syndrome, with a review of treatments for diffuse and circumscribed choroidal hemangiomas. Surv Ophthalmol. 2011;56:68-85.

20. Sullivan TJ, Clarke MP, Morin JD. The ocular manifestations of the Sturge-Weber syndrome. J Pediatr Ophthalmol Strabismus. 1992;29:349-56.

21. Thomas-Sohl KA, Vaslow DF, Maria BL. Sturge-Weber syndrome: a review. Pediatr Neurol. 2004;30:303-10.

22. Selbach JM, Posielek K, Steuhl KP, et al. Episcleral venous pressure in untreated primary open-angle and normal-tension glaucoma. Ophthalmologica. 2005;219:357-61.

23. Phelps CD, Armaly MF. Measurement of episcleral venous pressure. Am J Ophthalmol. 1978;85:35-42.

24. Olsen KE, Huang AS, Wright MM. The efficacy of goniotomy/ trabeculotomy in early-onset glaucoma associated with the Sturge-Weber syndrome. J AAPOS. 1998;2:365-8.

25. Tannous Z, Rubeiz N, Kibbi AG. Vascular anomalies: portwine stains and hemangiomas. J Cutan Pathol. 2010;37:88-95.

26. Ong T, Chia A, Nischal KK. Latanoprost in port wine stain related paediatric glaucoma. Br J Ophthalmol. 2003;87:1091-3.

27. Yang CB, Freedman SF, Myers JS, et al. Use of latanoprost in the treatment of glaucoma associated with Sturge-Weber syndrome. Am J Ophthalmol. 1998;126:600-2. 